\title{
TRANSPOSITION OF THE AORTA AND PULMONARY ARTERY DEMONSTRATED BY ANGIOCARDIOGRAPHY
}

\author{
BY \\ J. F. GOODWIN, R. STEINER, AND E. J. WAYNE \\ From the Department of Pharmacology and Therapeutics, University of Sheffield and the Department of \\ Radiology, Royal Infirmary, Sheffield. \\ Received February 22, 1949
}

Complete transposition of the great vessels is one of the less common congenital anomalies. Survival after birth is dependent upon the presence of some communication between the right and left sides of the heart that will permit crossing of the greater and lesser circulations and enable venous blood to reach the lungs.

The case described below is of particular interest because of the absence of diagnostic criteria afforded by the usual methods of clinical and radiological examination.

\section{CASE RePort}

D.T., aged 5 years, had been cyanosed since birth. He became very breathless on the slightest exertion and he frequently assumed the squatting position. There was no history of rubella during the mother's pregnancy.

Physical examination. The child was undersized, with marked cyanosis which was of equal intensity in both the upper and lower extremities. There was dyspncea at rest and marked clubbing of the fingers and toes. Examination of the cardiovascular system showed bulging of the præcordium and slight general cardiac enlargement. A harsh systolic murmur was heard down the left side of the sternum, and there was a pure pulmonary second sound. There was no thrill and no diastolic murmur. The femoral pulses and blood pressure were normal, and the lungs were clear.

The blood count showed a considerable degree of polycythæmia. The figures with the normal values at the age of 5 years for comparison were as follows: hæmoglobin 21 grams (12.6), red blood corpuscles 7.9 million per c.mm. (4.6), leucocytes 12,000 per c.mm., mean corpuscular diameter
$7 \cdot 3 \mu(7 \cdot 4)$, packed cell volume 63 per cent (37), mean corpuscular volume 80 c. $\mu$ (80), mean corpuscular hæmoglobin concentration 33 per cent (34).

Cardiography, using augmented unipolar leads in addition to standard leads, was performed and has been interpreted according to the criteria of Goldberger (1947) (Fig. 1). The standard limb leads show high voltage complexes and marked right axis deviation. The P waves in leads I and II and VF are of increased amplitude and duration (lead I, duration $0.11 \mathrm{sec}$., height $2 \mathrm{~mm}$; lead II, duration $0.12 \mathrm{sec}$, height $3 \mathrm{~mm}$.; lead VF, duration $0.11 \mathrm{sec}$, height $2.5 \mathrm{~mm}$.). The heart is vertical in position, since the unipolar left leg lead resembles lead V6 and therefore faces the epicardial surface of the left ventricle. There is also clockwise rotation of the heart round its long axis, because the unipolar left arm lead faces the cavity of the right ventricle. VL shows a small $R$ wave and a large $S$ wave and a negative $T$ wave and the præcordial leads show RS patterns.

There is no definite evidence of right ventricular strain, but this may be deduced from the presence of auricular hypertrophy. Goldberger states that if any of the unipolar extremity leads show $P$ waves with duration of $0.11 \mathrm{sec}$. or more and an amplitude of $2.5 \mathrm{~mm}$. or more, auricular hypertrophy may be suspected.

Radiography. A postero-anterior radiogram of the chest showed an enlarged heart with a prominent pulmonary conus and full root shadows; the lung fields showed congestive changes (Fig. 3).

Fluoroscopy and barium swallow revealed an enlarged heart, with an enlarged pulmonary conus, and very dilated pulmonary vessels with marked pulsation. The aorta appeared to be dextroposed. 

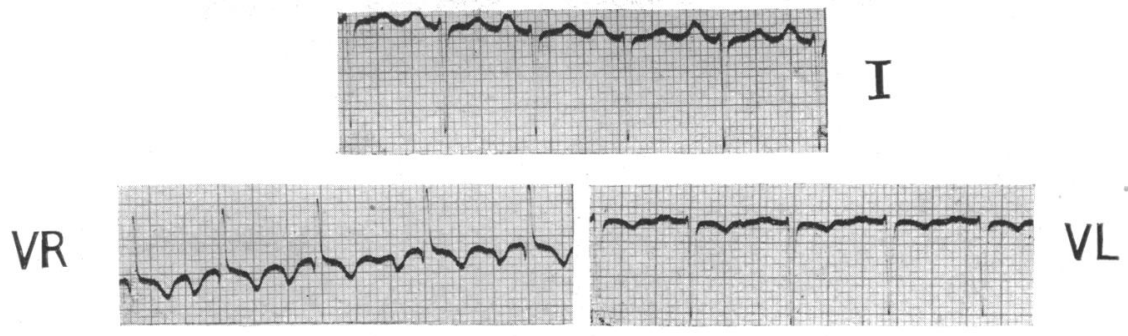

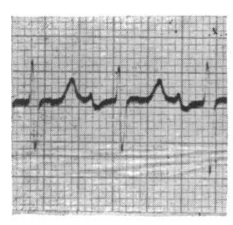

VI
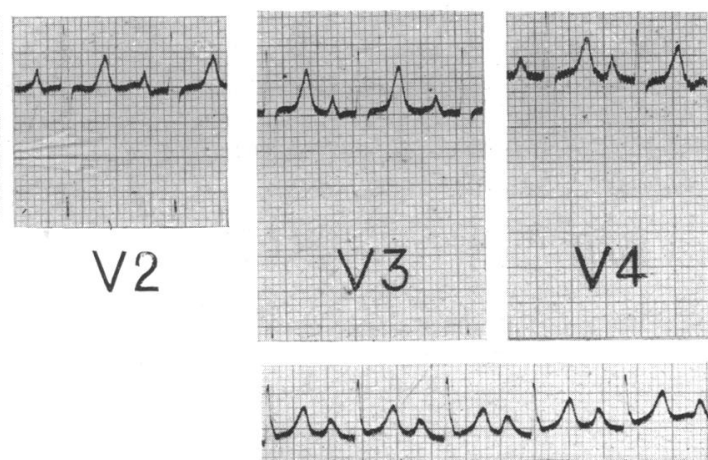

V4

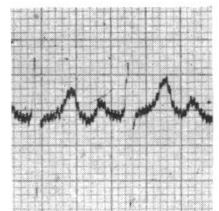

V5

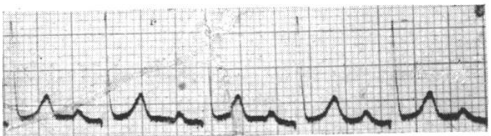

III

FIG. 1.-Standard and unipolar lead electrocardiograms. The heart is vertical with clockwise rotation round its long axis. Right auricular strain and indirect evidence of right ventricular strain are present. Limb lead $1 \mathrm{mv} .=1 \mathrm{~cm}$. Præcordial leads $1 \mathrm{mv} .=1 \cdot 5 \mathrm{~cm}$.

\section{ANGIOCARDIOGRAPHY}

Technique. Angiocardiography was carried out under general anæsthesia. Two injections of $40 \mathrm{ml}$. 70 per cent pyelosil were made into the antecubital vein of the right arm. In the first instance the patient was X-rayed in the right posterior oblique position lying supine, and in the second instance in the antero-posterior position lying on his side.

A rotating serial cassette changer was used with the following radiographic factors: 200 M.A. 72-74 K.V.P., 1/20th second exposure, at a target film distance of 36 inches. In both antero-posterior and oblique positions six exposures were made at one-second intervals starting at one second after the injection.

Oblique radiograms. On the two-second film (Fig. 3) good filling of the superior vena cava will be noted. The right auricle is clearly outlined and the reflux of contrast medium into the inferior vena cava is seen. The right ventricle is filled and enlarged and the interventricular septum is convex posteriorly. The ascending aorta and arch are outlined and a faint trace of contrast medium is visible in the left side of the heart just posterior to the right auricle. The pulmonary artery is outlined very faintly.

On the three-second film (Fig. 4) the right auricle is still outlined and the ventricle is more clearly shown, as well as the ascending aorta, aortic arch, and descending aorta. The innominate, and external and internal carotid arteries are also outlined. The left auricle and ventricle are fairly well delineated and the pulmonary artery can now be seen clearly demonstrated as a second arch below the aorta. On the subsequent films most of the contrast medium is seen scattered throughout the pulmonary vascular bed and the cardiac chambers are not clearly differentiated.

Antero-posterior radiograms. In the two-second film (Fig. 5A and B) the right auricle and ventricle are clearly demonstrated. The ascending aorta is seen to arise from the right ventricle. The 


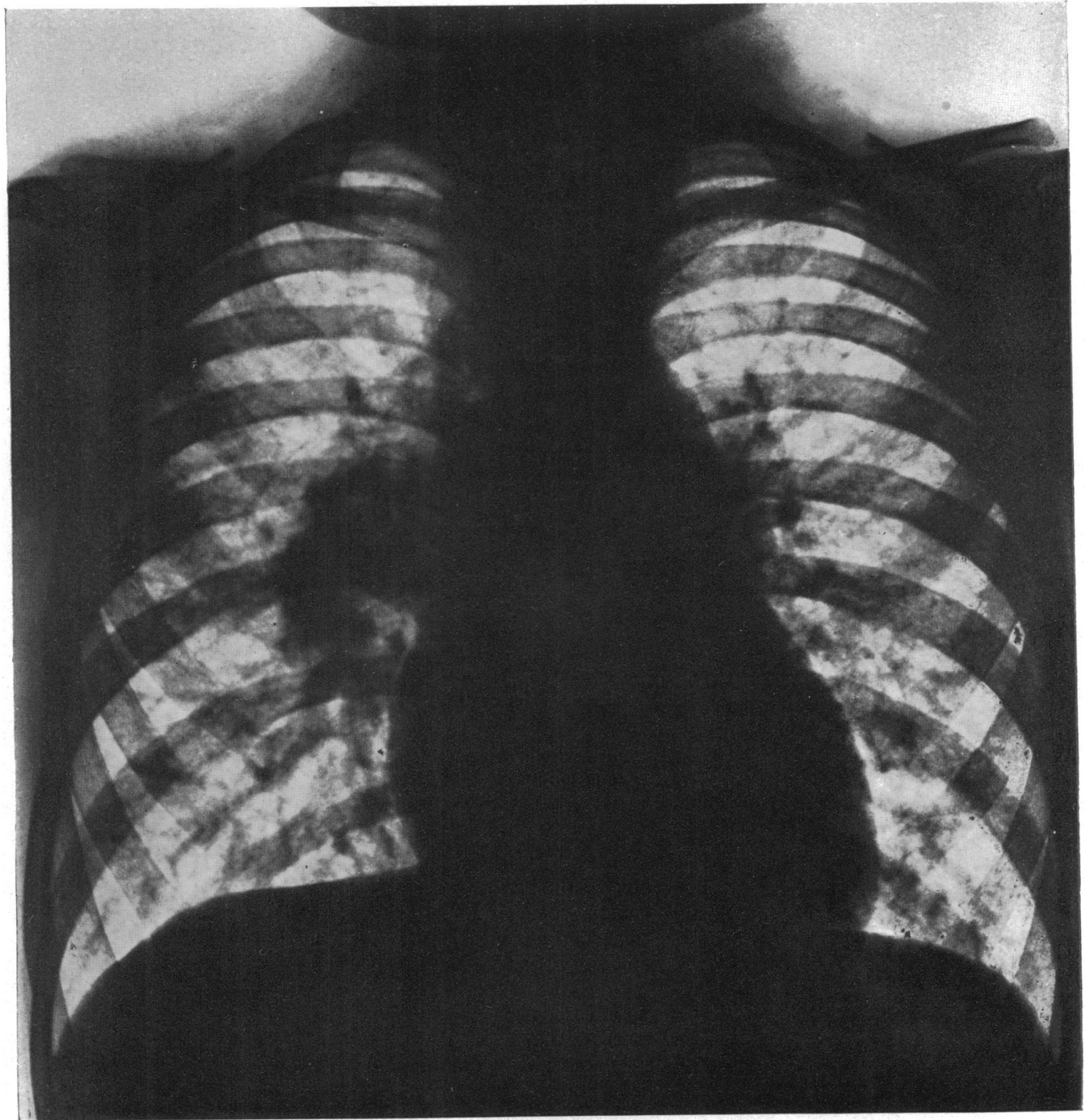

Fig. 2.-Postero-anterior 6-ft. film of the chest.

innominate artery, the right carotid and the internal carotid arteries are shown and some contrast medium has entered the left ventricle. On the three-second film (Fig. 6) the left side of the heart is now filled with contrast medium. The aorta is still shown; the pulmonary artery and the main pulmonary branches are partly demonstrated. As in the case of the oblique radiograms subsequent films did not reveal any details of the cardiac chambers as most of the contrast 'medium had entered the pulmonary vascular bed and partly left the heart.

The radiographic examination quite clearly demonstrated the transposition of the aorta and pulmonary artery. The septal defect itself is not shown, but the rapid filling of the left side of the heart on the two- and three-second films is very suggestive of the presence of such a defect. It is 

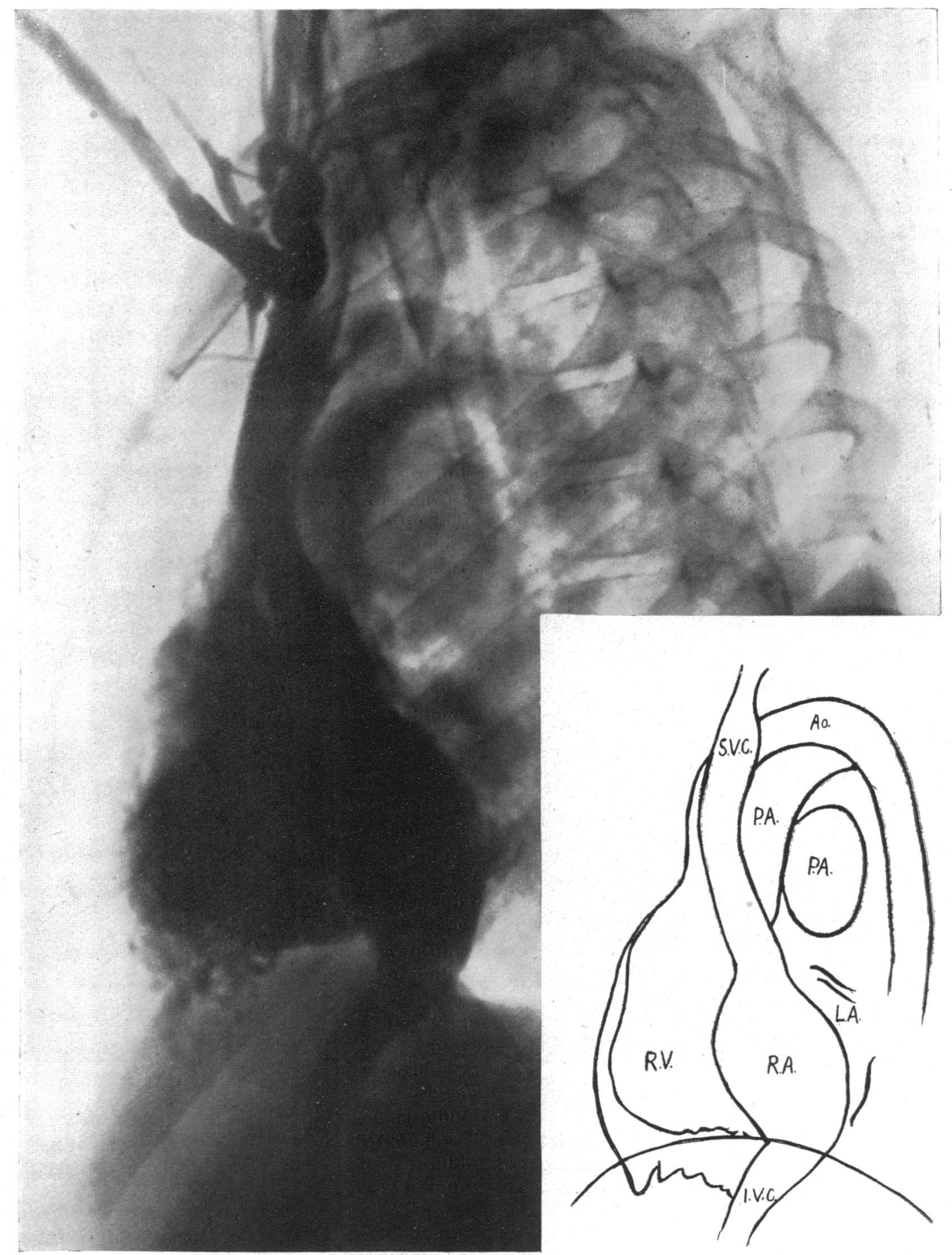

A

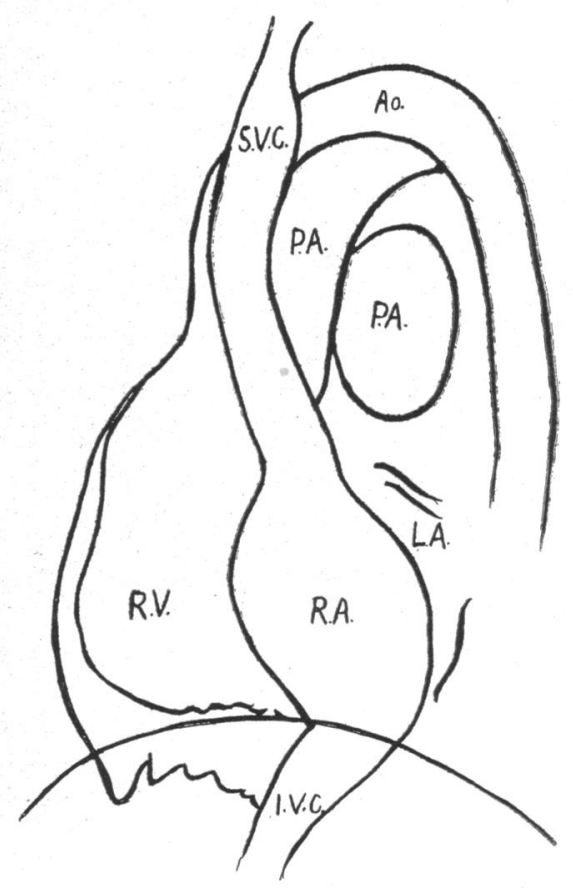

B

Fig. 3.-Angiocardiogram at 2 seconds. Patient in the right posterior oblique position. The right auricle and ventricle are demonstrated as well as the pulmonary artery and aorta.

(B) (Inset) Diagram of Fig. 4A. S.V.C.=Superior vena cava I.V.C. = Inferior vena cava

R.A. = Right auricle

L.A. $=$ Left auricle

R.V. = Right ventricle

P.A. = Pulmonary artery

Ao. $=$ Aorta 


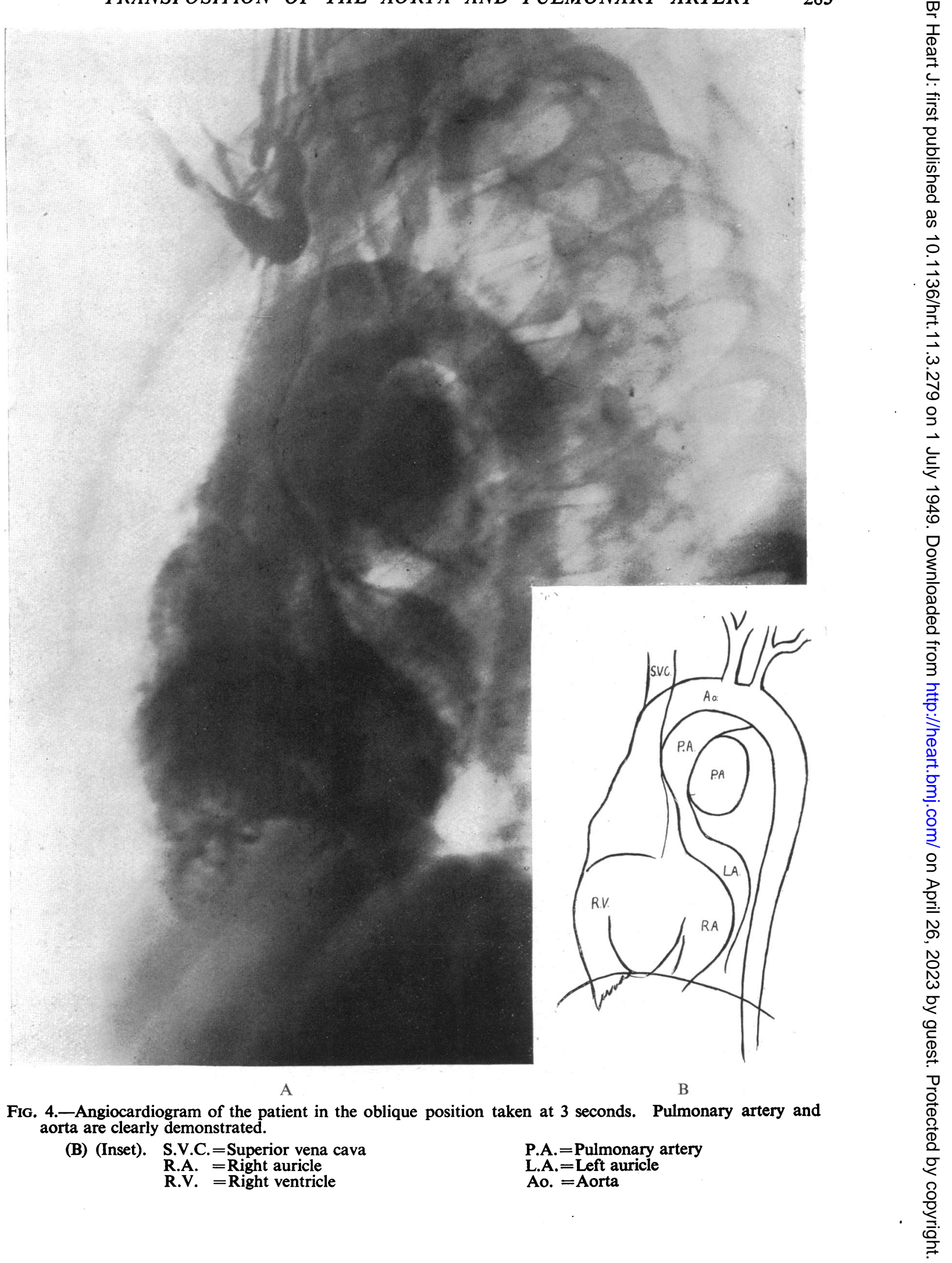




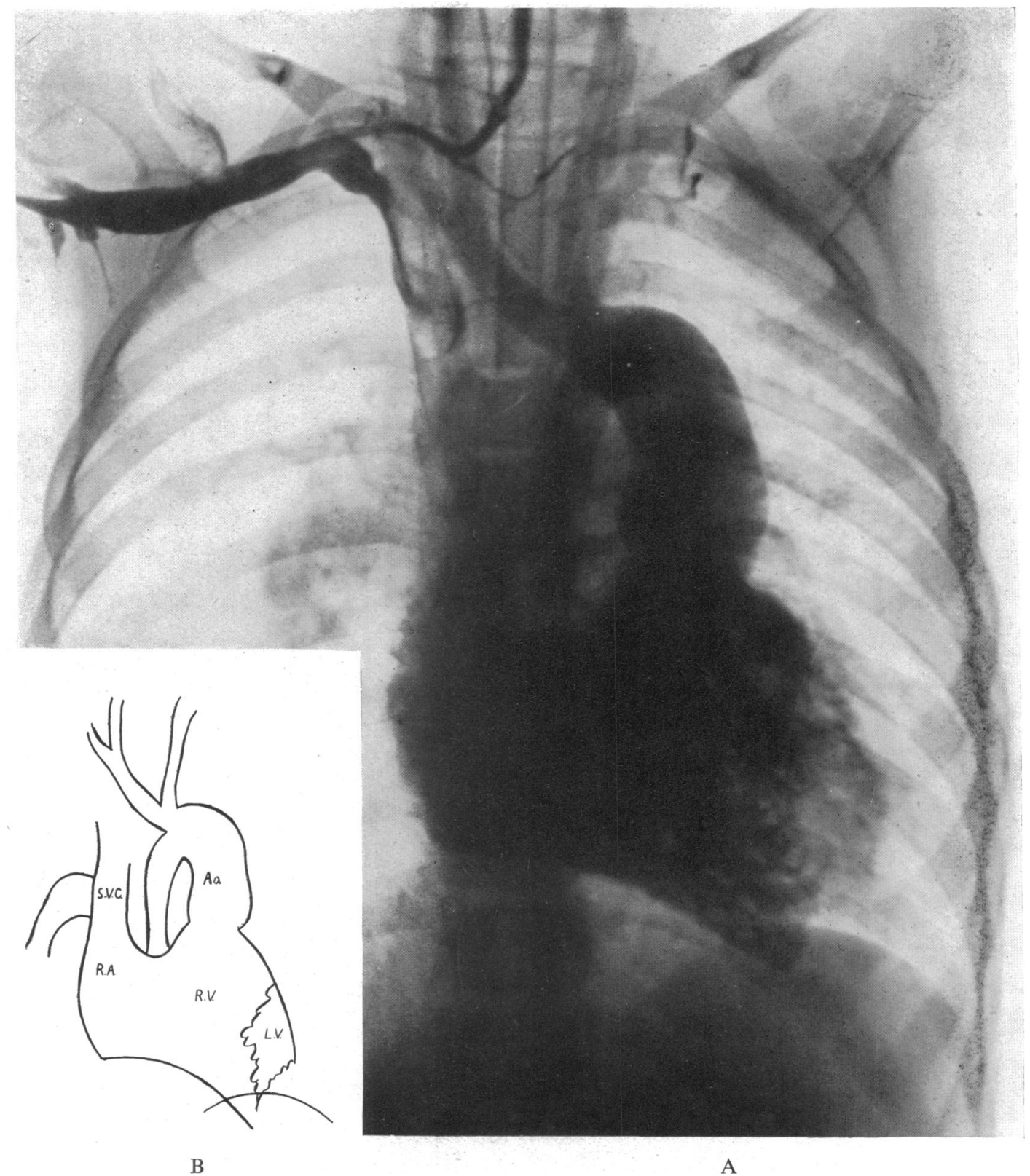

FIG. 5.-Angiocardiogram, at 2 seconds, of the patient in the antero-posterior position. Right auricle and ventricle are clearly demonstrated. The aorta is seen to rise from the right ventricle.

(B) (Inset). S.V.C. = Superior vena cava
R.A. $=$ Right auricle
R.V. = Right ventricle
L.V. = Left ventricle
Ao. = Aorta 


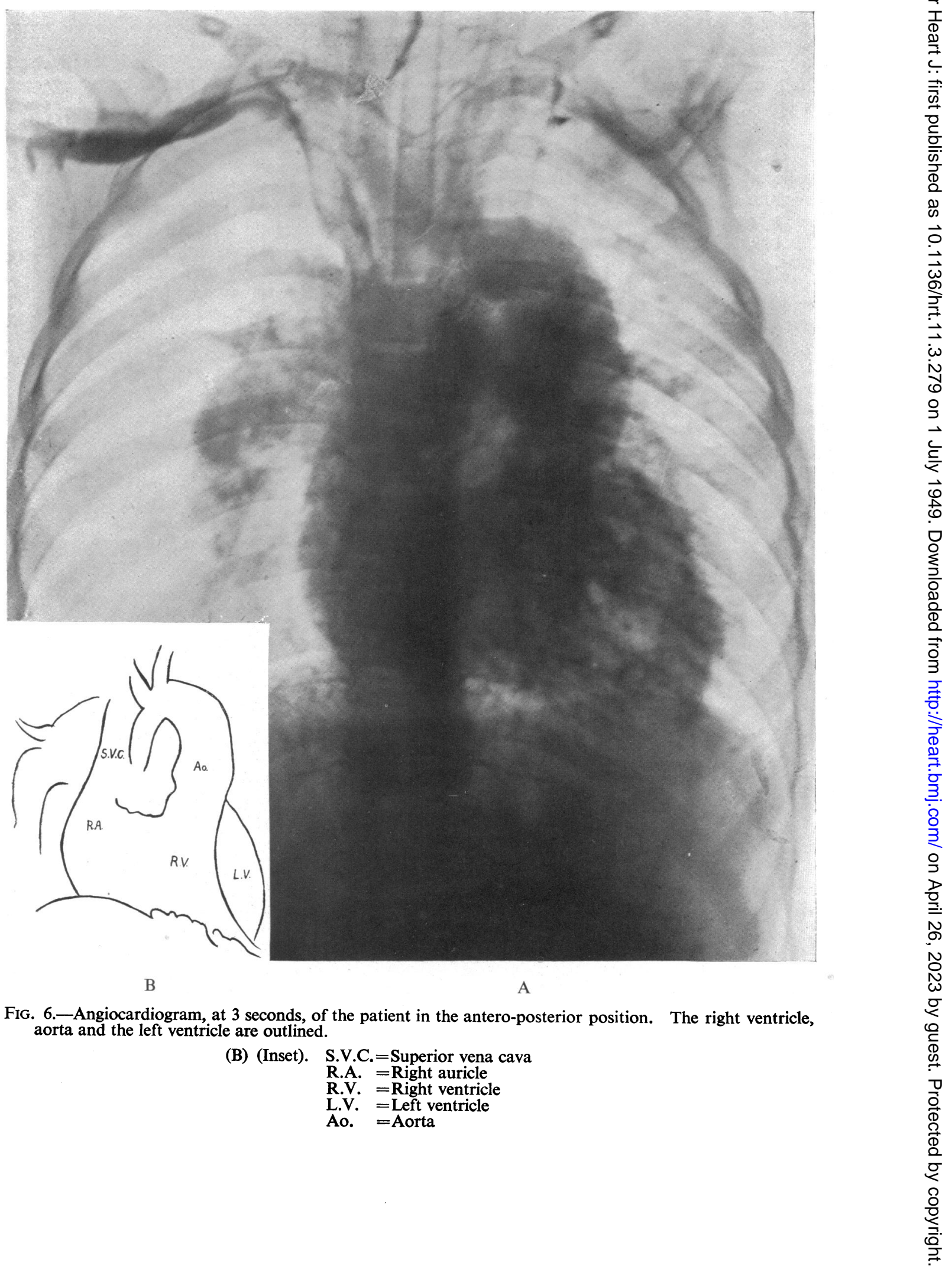


impossible to decide if this defect is auricular or ventricular.

Cardiac catheterization was not undertaken as it was felt that it would yield no further useful information.

\section{Differential Diagnosis}

The history of cyanosis from birth and the physical signs in this case suggested either transposition of the aorta and pulmonary artery with an associated widely patent septum, tricuspid atresia, single ventricle, or Fallot's tetrad with extreme pulmonary stenosis. Electrocardiography did not reveal the gross right or left heart strain that would have been expected in pulmonary and tricuspid atresia respectively. Taussig (1945) describes a characteristic cardiac contour on fluoroscopic examination in cases of transposition of the great vessels. Since the aorta lies further to the right and in front and the pulmonary artery further to the left and behind, the shadow at the base of the heart is the reverse of normal, being wider in the left anterior oblique view and narrower in the antero-posterior view. In the latter position the shadow of the pulmonary conus is usually absent and a rhythmical change in size of the right auricle and ventricle, due to frequent reversal of the shunt, is seen. In our case no such distinctive contour or rhythmical alteration was observed, and a pulsating shadow noted in the region of the pulmonary conus was proved by angiocardiography to be the aorta.

\section{Discussion}

Complete transposition of the aorta and pulmonary artery may be accompanied by patencies of the normal fotal pathways, the foramen ovale, and the ductus arteriosus, or by additional septal defects. As Taussig (1947) points out, the larger the communication between the two sides of the heart, the longer will be the period of survival. When the ductus arteriosus or foramen ovale alone are patent, survival for more than a few hours or days does not occur, but infants who in addition have widely patent interventricular septa may survive for much longer. Taussig considers that even in cases where both septa are widely patent survival is rarely possible beyond the age of eighteen months. Hanlon and Blalock (1948) collected 123 cases of complete transposition with associated abnormalities and found the average duration of life was nineteen months. Six patients lived ten years or longer, but the average age at death in the other 117 patients was five and a half months. Twelve cases had an interventricular septal defect without other abnormalities, and these patients lived for a mean period of four years and one month. The association of a patent foramen ovale with patent interventricular septum (19 cases) increased the survival to four years nine months, but the association of other defects decreased the duration of life. Of Kato's (1930) 86 cases, 16 had defects of both septa, the average survival time of these 16 being five and a half years. But the inclusion of 2 patients aged nineteen and fifty-six years respectively among these 16 cases presents a falsely optimistic picture. It appears, therefore, that complete transposition is unlikely to be compatible with survival after the age of four years, even when a patent interventricular septum is present.

\section{SUMmary}

A case of complete transposition of the aorta and pulmonary artery is described in a child five years of age showing intense cyanosis and great breathlessness on exertion. The condition was clearly revealed by angiocardiography and could not have been detected by the usual clinical and radiological methods. A survey of the literature shows that survival beyond the age of four and a half years is very rare in this condition.

We wish to thank Professor R. S. Illingworth for permission to publish the details of this case, Dr. J. Wilkie and Dr. C. W. Lawson for their help and cooperation, and Mr. J. Coombs for technical assistance.

\section{REFERENCES} Goldberger, E. Unipolar Lead Electrocardiography. Kato, K. (1930). Amer. J. Dis. Child., 39, 363.

Hanlon, C. R., and Blalock, A. (1948). Ann. Surg.

Taussig, H. B. Congenital Malformations of the Heart. 127, 385 . New York, 1947. 